\title{
Deuda y quiebra familiar en tiempos de confinamiento por COVID-19: caso provincia de Jaén
}

\author{
Victor Hugo Puican Rodríguez
}

Universidad Nacional de Jaén, Perú

\begin{abstract}
Resumen
Este artículo tuvo como objetivo general determinar el efecto que tuvo el confinamiento por COVID-19 en la deuda y quiebra de las familias de la ciudad de Jaén. Se manejó un enfoque cuantitativo, no experimental, con una muestra de 123 hombres y mujeres mayores de edad que radican en la ciudad de Jaén. Asimismo, se utilizó la técnica de la encuesta y como instrumento el cuestionario. Los resultados obtenidos evidencian que el impacto del confinamiento social ha sido devastador en la economía familiar, ya que 107 de los encuestados manifestaron que perdieron su empleo, 113 mantienen deudas a la fecha con alguna entidad financiera, 118 de los encuestados manifestaron que son los únicos que aportan económicamente a su familia. Con todo lo anterior, se concluye

que la pérdida del empleo por el aislamiento social decretado por el Gobierno ha generado un incremento masivo de la pobreza extrema en la ciudad de Jaén porque los habitantes perdieron su trabajo, mantienen deudas vencidas con entidades financieras, donde ninguna ha brindado facilidad alguna ante las deudas vencidas ni dejó de cobrar intereses. Y como suma, a pesar de estas dificultades, no han recibido el apoyo económico que el Gobierno entregó en tiempos de pandemia.
\end{abstract}

Palabras clave: confinamiento, economía, pobreza, desempleo, ciudadano. 


\title{
Debt and family bankruptcy in times of confinement due to COVID-19: case of the province of Jaen
}

\begin{abstract}
The general objective of this article was to determine the effect of COVID-19 confinement on the debt and bankruptcy of families in the city of Jaén. A quantitative, non-experimental approach was used with a sample of 123 men and women of legal age living in the city of Jaén. The survey technique was used and the questionnaire was used as an instrument. The results obtained show that the impact of social confinement has been devastating for the family economy, since 107 of those surveyed stated that they lost their jobs, 113 are still in debt with some financial entity, 118 of those surveyed stated that they were the only ones who contributed economically to their family. With all of the above, it is concluded that the loss of employment due to the social isolation decreed by the Government has generated a massive increase in extreme poverty in the city of Jaén because the inhabitants lost their jobs, maintain overdue debts with financial entities, where none has provided any facility to the overdue debts or stopped charging interest. And in addition, in spite of these difficulties, they have not received the economic support that the Government provided in times of pandemic.
\end{abstract}

Keywords: confinement, economy, poverty, unemployment, citizen.

\section{Dívida e família familiar em tempos de confinamento por COVID-19: caso da província de Jaén}

\begin{abstract}
Resumo
O objectivo geral deste artigo era determinar o efeito do confinamento COVID-19 sobre a dívida e falência de famílias na cidade de Jaén. Foi utilizada uma abordagem quantitativa, não experimental, com uma amostra de 123 homens e mulheres com idade legal a viverem na cidade de Jaén. Da mesma forma, foi utilizada a técnica de inquérito e o questionário foi utilizado como um instrumento. Os resultados obtidos mostram que o impacto do confinamento social tem sido devastador na economia familiar, uma vez que 107 dos inquiridos declararam ter perdido o seu emprego, 113 mantêm dívidas até à data com alguma entidade financeira, 118 dos inquiridos declararam ser os únicos que contribuíram economicamente para a sua família. Com

tudo isto, pode concluir-se que a perda de emprego devido ao isolamento social decretado pelo governo gerou um aumento maciço da pobreza extrema na cidade de Jaén porque os habitantes perderam os seus empregos, mantêm dívidas vencidas com entidades financeiras, onde nenhum deles providenciou qualquer facilidade para as dívidas vencidas ou deixou de cobrar juros. E além disso, apesar destas dificuldades, não receberam o apoio económico que o governo lhes deu em tempos de pandemia.
\end{abstract}

Palavras-chave: confinamento, economia, pobreza, desemprego, cidadão. 


\section{Introducción}

El aislamiento social decretado por el gobierno en la segunda quincena de marzo del 2020, "ha implicado un choque económico tanto de oferta como de demanda" (Clavellina \& Domínguez, 2020, p. 1), lo cual ha ocasionado que diferentes empresas se acogieran a la suspensión perfecta de labores por la "crisis económica ocasionada perjudicando tanto a los trabajadores como a los propietarios de capital" (Rodríguez, 2021, p. 251). Como consecuencia, la peor parte recayó sobre los hogares de los trabajadores despedidos, lo que afectó directamente en la economía familiar, ya que no contaban con ingresos mensuales para cubrir las necesidades básicas del hogar.

Asimismo, García y Almeida (2021) manifiestan que el Covid-19 ha ocasionado un crecimiento de la pobreza y pérdidas económicas en las familias debido al quiebre de las empresas y la pérdida de los puestos de trabajo. Ello ocasionó que el nuevo grupo desempleado no cumpliera con sus pasivos corrientes y no corrientes asumidos por concepto de créditos de consumo con las entidades financieras, con el pago de alquileres y la compra de alimentos de primera necesidad.

De acuerdo a la Comisión Económica para América Latina y el Caribe (CEPAL, 2020), ante la caída del 5,3\% del PIB y el aumento del desempleo en América Latina, la pobreza aumentaría al menos en 4,4 puntos porcentuales, lo que provocaría a su vez el aumento de la pobreza extrema en 2,6 puntos porcentuales; en consecuencia, un total de 83,4 millones de personas se verían afectadas.

Debido a la falta de ingresos, muchas familias han optado por no obtener ningún crédito financiero ni usar sus escasos recursos económicos en gastos que no son prioridad. Es aquí donde las entidades financieras se ven afectadas, pues cuentan con demasiada liquidez. De la misma forma, las mismas entidades deben buscar alternativas de inversión debido al deterioro de las políticas crediticias, producto del débil grado de competitividad con el que operan tanto a nivel nacional como internacional (Martínez, Bucio, \& Ortíz, 2021).

Según el Banco Central de Reserva del Perú (BCRP, 2020), la economía peruana experimentó en el 2020 la mayor caída en los últimos 100 años. Si bien en el 2021 nuestra economía presentó una fuerte recuperación, se vio afectada nuevamente por el nuevo aislamiento que dictaminó el gobierno, y podría verse aún más afectada por el contexto electoral, con lo cual, según cifras oficiales, podría contraerse el PBI en un $12,7 \%$.

De acuerdo al Instituto Nacional de Estadística e Informática (INEI, 2019), en el 2018 la población económicamente inactiva incrementó, con respecto al año 2017, en $1,9 \%$, por lo que se proyectó para el 2020 un incremento del 2,2\% y de 2,5\% para el 2021. Sin embargo, estos niveles incrementaron por el impacto del COVID-19 en nuestro país: la tasa de desempleo llegó hasta el 12,70\%, y para el 2021 se proyecta un $11 \%$, donde los mayores afectados son varones (Sociedad de Comercio Exterior del Perú, 2020).

El Fondo Monetario Internacional (FMI, 2020), manifestó que, debido a la fuerte expansión del COVID-19, la economía global está atravesando la crisis más severa en 
los últimos 90 años, con lo cual proyecta que para octubre del 2020 la economía caería en $4,4 \%$. La economía peruana no ha sido la excepción, ya que se ha visto afectada por shocks negativos sobre la oferta de bienes y servicios, por las interrupciones en la cadena de suministros y reducción de la producción en alimentos de primera necesidad, y la demanda de bienes y servicios se vio afectada por el despido masivo de trabajadores; todo esto tendría como consecuencia un recorte en los ingresos económicos de muchas familias a nivel nacional (SBS, 2020).

A criterio de Herrera y Reys (2020), a partir de un estudio de la evolución de los ingresos de las familias, más de la mitad de los hogares pobres perdieron la gran mayoría de sus ingresos durante la cuarentena, mientras que solo uno de cinco hogares mantiene sus ingresos sin variación o con una ligera disminución. Con ello se concluye que el COVID-19 impactó de manera drástica en las familias peruanas.

\section{Marco teórico}

Una vez planteado el contexto de crisis económica que atravesamos por el COVID-19, revisaremos algunos autores que nos permitirán sustentar nuestra visión sobre los efectos de la pandemia en la economía familiar.

En primer lugar, Narváez (2020), a través de su artículo científico -un estudio descriptivo, correlacional, no experimental-, nos demuestra que, hasta el mes de junio del 2020, la morosidad global o cartera de alto riesgo de las entidades financieras ascendía a 6604 millones de soles, donde el 33,15\% del total eran colocaciones y había una tasa de morosidad del 45,37\%. A partir de lo anterior se puede concluir, por un lado, que la recuperación de la economía no será a corto plazo, y por otro, la deuda diferida será mayor en ese momento y probablemente resulte impagable.

Luego, a partir de la revisión del artículo de Lozano, Lozano y Robledo (2020), se extrae que la alteración de las cadenas de producción provoca que las empresas prescindan o despidan a sus trabajadores, con lo que decrece la demanda final; en paralelo, la demanda de materias primas disminuye el consumo productivo, por lo cual en el periodo mayo-junio 2020 la tasa de desempleo alcanzó el 13,3\% a nivel nacional, en comparación al 3,8\% de diciembre del 2019. Con todo lo anterior se ratifica lo visto en los anteriores párrafos: el entorno familiar se ha visto seriamente afectado no solo porque se deben cumplir con medidas radicales de distanciamiento social, sino también por el desempleo y la pérdida de los ingresos familiares per cápita.

Como tercer texto tenemos el de Parada y Zambrano (2020), quienes, a través de las entrevistas y de un enfoque cualitativo de tipo fenomenológico, obtuvieron como resultados que, a raíz del aislamiento, las personas no habían podido trabajar por ser independientes o por haber quedado desempleadas, lo cual generó la reducción o pérdida de sus ingresos, e incluso, la desaparición de la única fuente económica familiar. A partir de lo anterior, el estudio concluye que las familias afrontan graves problemas económicos porque la única fuente de ingresos que tenían era su trabajo, 
donde en algunos casos se debe a que las empresas optaron por la suspensión perfecta de labores.

Por último, Cuenca, Osorio, Pastor, Peña, y Torres (2020) formularon cómo la cuarentena y el COVID-19 tuvieron efectos sobre los aspectos económicos y de salud. Para ello usaron un enfoque cuantitativo de tipo descriptivo y no experimental, con una muestra de 1064 peruanos en todo el país. De la investigación se obtuvo que, en cuanto a la situación económica, el 57,3\% refiere que ha presentado muchos o algunos problemas económicos para comprar productos de primera necesidad; el $69,8 \%$ manifiesta que tiene alimentos en la actualidad para menos de siete días; y el $56,8 \%$ tiene dinero suficiente para menos de dos semanas. Asimismo, el 79,5\% refiere no que ha sido beneficiado con algún bono o subsidio del estado, y el 65,8\% declara que los bonos o subsidios son poco para poder adquirir lo básico. A partir de lo anterior se concluye que existen problemas económicos para comprar alimentos y medicinas, los alimentos solo alcanzan para una semana, el dinero es escaso por la falta de trabajo, los bonos son pocos para adquirir lo básico y la mayor parte de la familia no ha sido beneficiada por ningún bono.

\section{Metodología}

Una vez delimitado el marco teórico, el presente trabajo procederá a señalar su metodología, la cual consistirá en un enfoque cuantitativo y de diseño no experimental. De la misma manera se utilizó como técnica la encuesta. Esta fue aplicada a 123 ciudadanos que radican en la ciudad de Jaén, entre los 18 a 51 años de edad y de ambos sexos, quienes tuvieron que responder un cuestionario compuesto por 09 ítems. Dicho cuestionario, titulado "Cuestionario sobre deuda y crisis familiar: caso provincia de Jaén", y el cual será la fuente base para la presentación de nuestras tablas, fue elaborado en Google formulario y aplicado entre el 05 de febrero y el 07 de marzo del 2021. Se configuró a una respuesta por usuario, y fue difundido por redes sociales como WhatsApp, Facebook y Messenger.

\section{Resultados}

Después de haber aplicado la encuesta a 123 ciudadanos de la ciudad de Jaén, de los cuales 63 tienen entre 26 a 35 años, 28 entre 18 a 25 años, 25 entre 36 a 50 años y 7 más de 51 años, y de los cuales 66 de los encuestados fueron mujeres y 57 hombres, tuvimos los siguientes números: 


\section{Tabla 1: ¿Se encontraba laborando antes del aislamiento social decretado por el Gobierno?}

\begin{tabular}{|c|c|}
\hline Etiquetas de fila & Cuenta de 1. Género: \\
\hline NO & 14 \\
\hline SI & 109 \\
\hline Total general & $\mathbf{1 2 3}$ \\
\hline
\end{tabular}

Fuente: Elaboración propia

\section{Figura 1:}

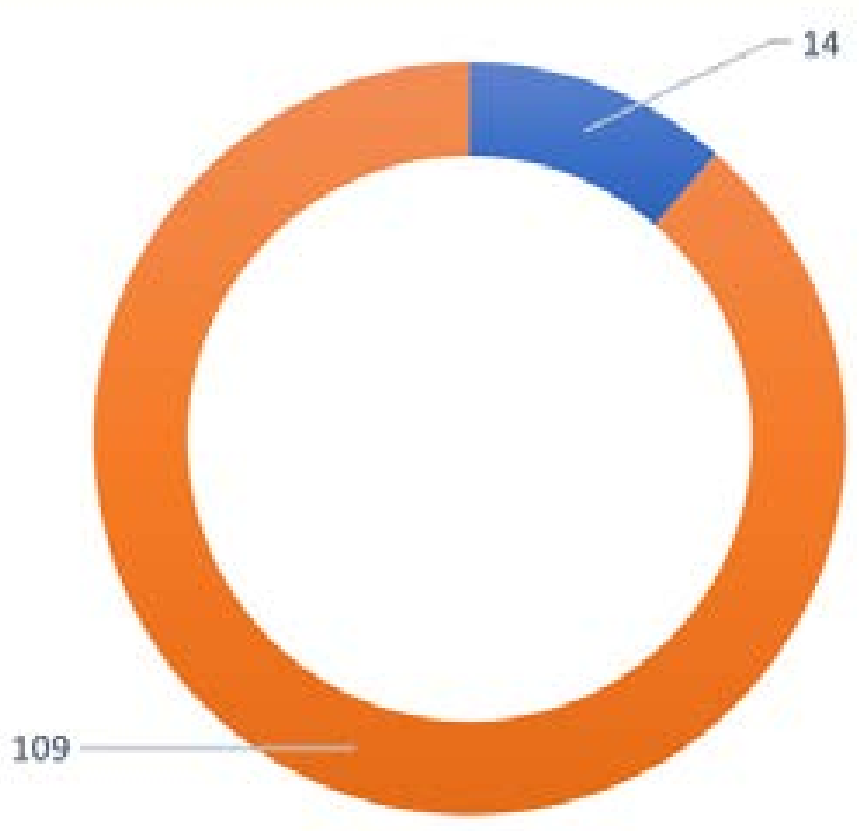

$=$ NO $=S 1$

Fuente: Elaboración propia

De acuerdo a los resultados obtenidos en la tabla y figura 1, 109 manifestaron que se encontraban laborando antes del inicio del aislamiento social, y solo 14 de los encuestados no se encontraban ejerciendo algún empleo. 
Tabla 2: ¿El aislamiento social decretado desde la segunda quincena de marzo del 2020, causó que usted perdiera su empleo?

\begin{tabular}{|c|c|}
\hline Etiquetas de fila & Cuenta de 1. Género: \\
\hline NO & 16 \\
\hline SI & 107 \\
\hline Total general & $\mathbf{1 2 3}$ \\
\hline
\end{tabular}

Fuente: Elaboración propia

\section{Figura 2:}

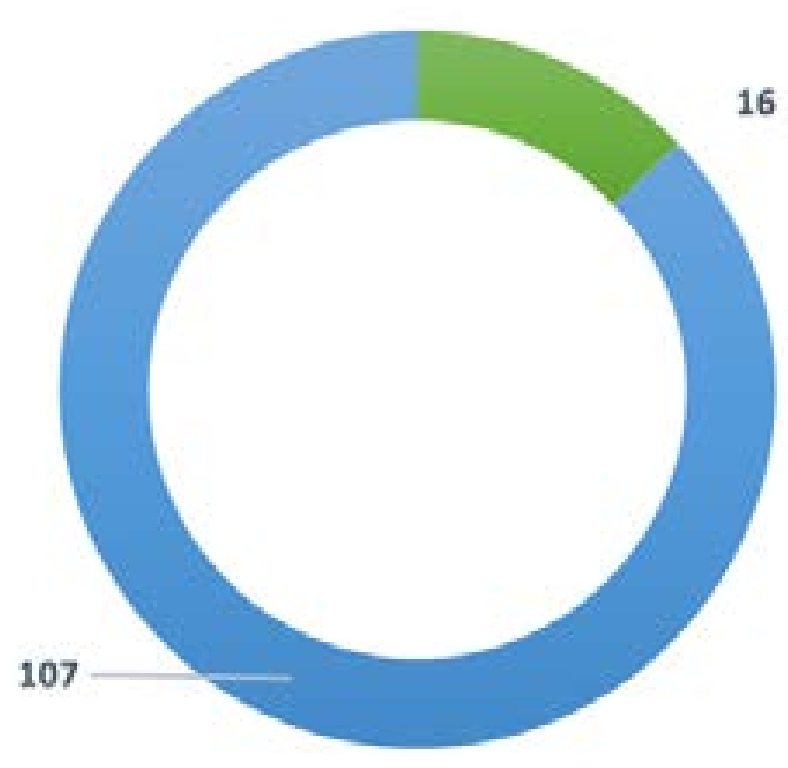

Fuente: Elaboración propia

De los 123 encuestados, 107 de ellos perdieron su trabajo por motivos del aislamiento social y solo 16 de los encuestados continuaron laborando pese al aislamiento social. Con ello se evidencia que el cierre de las actividades empresariales ha impactado con fuerza en la economía de las familias, lo cual les privó de percibir ingresos económicos y afectó considerablemente el bienestar de cada uno de los integrantes de la familia. 


\section{Tabla 3: ¿Usted mantiene deuda vencida con alguna entidad financiera?}

\begin{tabular}{|c|c|}
\hline Etiquetas de fila & Cuenta de 1. Género: \\
\hline NO & 10 \\
\hline SI & 113 \\
\hline Total general & $\mathbf{1 2 3}$ \\
\hline
\end{tabular}

Fuente: Elaboración propia

\section{Figura 3:}

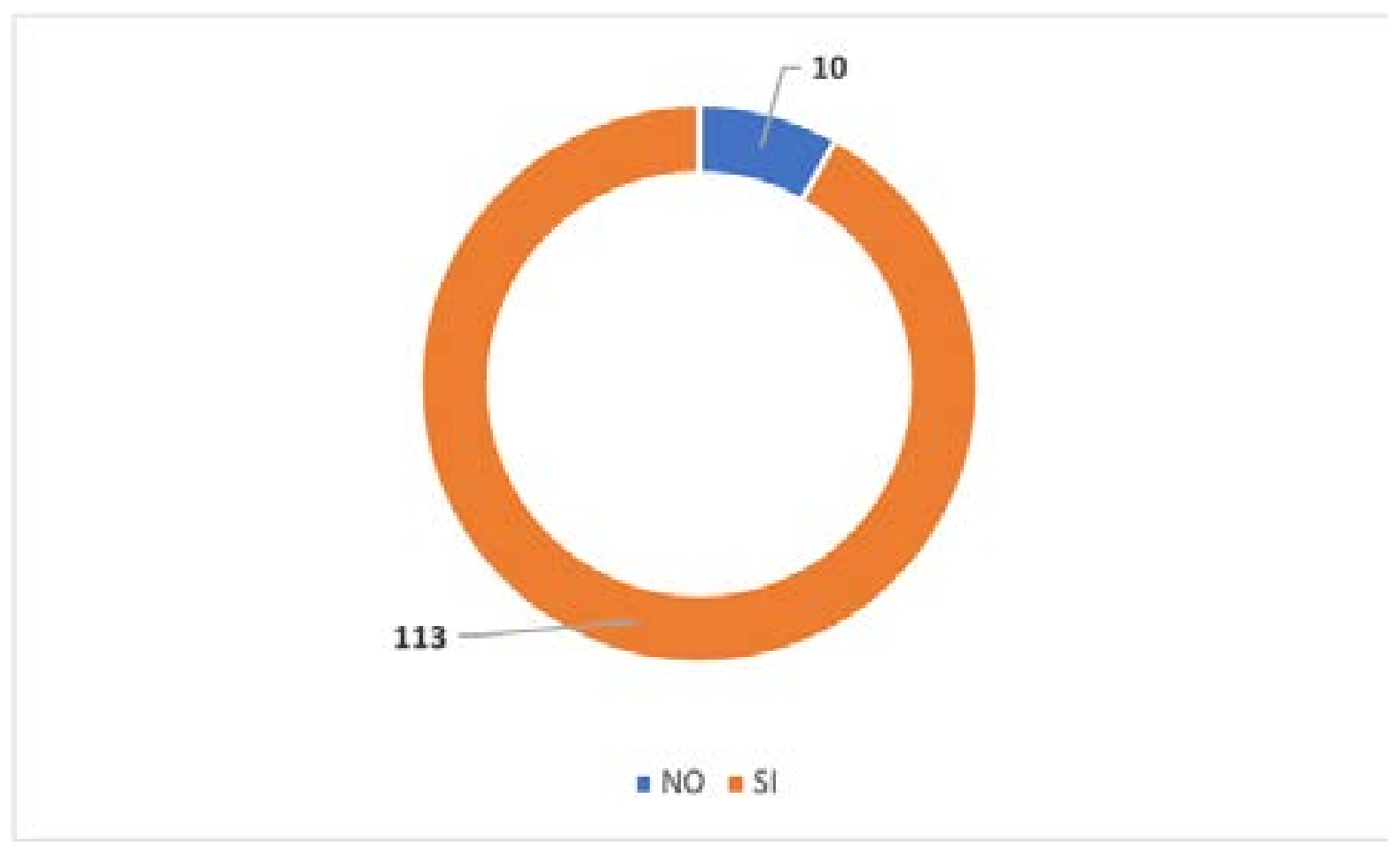

Fuente: Elaboración propia

De los 123 encuestados, 113 de ellos mantienen una deuda vencida con alguna entidad financiera, y solo 10 no mantienen deudas vencidas con ninguna institución bancaria. Como se puede apreciar la gran mayoría de encuestados mantienen una deuda por préstamo con alguna entidad financiera, la cual no podrán pagar porque no mantienen una relación laboral activa con alguna organización. 
Tabla 4: ¿Usted es el único que aporta económicamente a la familia?

\begin{tabular}{|c|c|}
\hline Etiquetas de fila & Cuenta de 1. Género: \\
\hline NO & 05 \\
\hline SI & 118 \\
\hline Total general & $\mathbf{1 2 3}$ \\
\hline
\end{tabular}

Fuente: Elaboración propia

\section{Figura 4:}

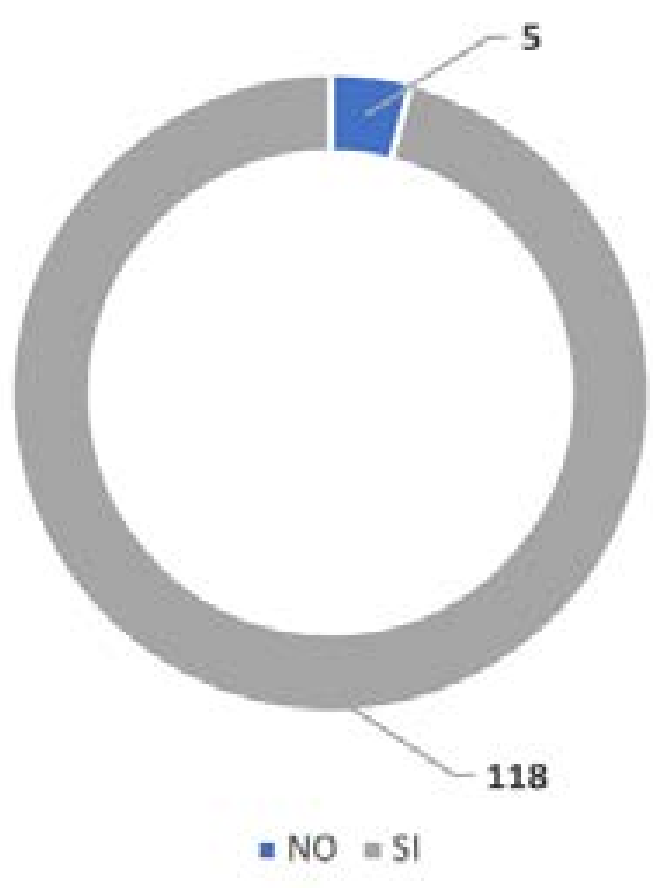

Fuente: Elaboración propia

De los 123 encuestados, 118 manifestaron que son los únicos que aportan económicamente en la familia, lo cual afecta considerablemente la situación económica familiar, aumenta la pobreza extrema en la ciudad de Jaén y afecta la salud de los niños y de los padres en una de las familias. 
Tabla 5: ¿Su familia ha sido beneficiada con algún bono que el Estado entregó en tiempos de pandemia?

\begin{tabular}{|c|c|}
\hline Etiquetas de fila & Cuenta de 1. Género: \\
\hline NO & 89 \\
\hline SI & 34 \\
\hline Total general & 123 \\
\hline
\end{tabular}

Fuente: Elaboración propia

\section{Figura 5:}

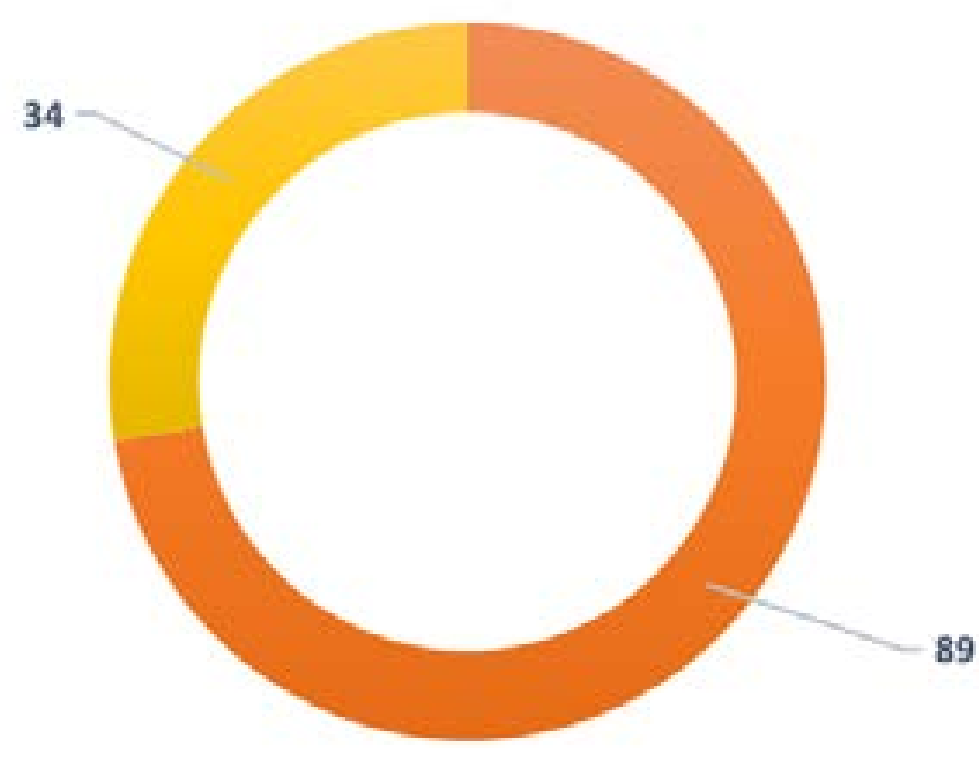

Fuente: Elaboración propia

De los 123 encuestados, 89 manifestaron que no han recibido ningún bono económico por parte del Estado y solo 34 mencionaron que sé recibieron un bono. Por lo tanto, la falta de trabajo, las deudas, enfermedades y la carencia de un bono por parte del Gobierno complica la situación de estas personas.

\section{Conclusiones}

- La cuarentena ha afectado a todos los estratos económicos, y la pérdida del empleo por el aislamiento social decretado por el Gobierno ha generado un 
incremento masivo de la pobreza extrema en el país, pues vemos que personas que pertenecían a la clase medía han retrocedido a la clase pobre.

- La falta de una lista actualizada de todos los peruanos ha ocasionado que los bonos económicos no lleguen a las personas que en realidad lo necesitan. Debido a ello, vemos mayor desigualdad, un aumento de la crisis familiar y una carencia de alimentos de primera en los hogares.

- Las entidades financieras no apoyaron en prorratear las cuotas vencidas sin interés adicional. Esto generó que algunas familias vendieran algunos bienes y quedaran en la bancarrota para poder cumplir con estos pagos.

\section{Referencias bibliográficas}

BCRP. (2020). Reporte de Inflación: Panorama actual y proyecciones macroeconómicas 2020-2021 (Informe setiembre 2020). Recuperado de https://www.bcrp.gob.pe/ docs/Publicaciones/Reporte-Inflacion/2020/setiembre/reporte-de-inflacionsetiembre-2020.pdf

CEPAL. (2020). El desafío social en tiempo del COVID - 19 (Informe $\mathrm{n}^{\circ} 3$ ). Recuperado de https://repositorio.cepal.org/bitstream/handle/11362/45527/S2000325_ es.pdf?sequence $=5 \&$ is Allowed $=y$

Clavellina, J., \& Domínguez, M. (2020). Implicaciones económicas de la pandemia por COVID-19 y opciones de política. notasestratégicas, 81, 1-11. Recuperado de http://www.bibliodigitalibd.senado.gob.mx/bitstream/handle/123456789/4829/ NE_coronavirus_implicaciones\%20econ\%c3\%b3micas\%20_010422020. pdf? sequence $=1$ \&isAllowed $=y$

Cuenca, C., Osorio, M. L., Pastor, J. L., Peña, G. D., \& Torres, L. S. (2020). Aspectos económicos y de salud en tiempos de cuarentena por COVID - 19 en población peruana, año 2020. Revista Facultad Medicina Humana, 20(4), 630-639. Recuperado de http://www.scielo.org.pe/pdf/rfmh/v20n4/2308-0531-rfmh-20-04-630.pdf

FMI. (2020). Crecimiento económico global: variación porcentual anual.

García, S., \& Almeida, P. (2021). Ecuador: Situación macroeconómica en 2020 y perspectivas 2021. Colegio de Economistas de Pichincha, 1-11. Recuperado de https://colegiodeeconomistas.org.ec/wp-content/uploads/2021/01/PerspectivasMacroeconomicas-Ecuador-2020-y-2021.pdf

Herrera, T. \& Reys, A. (2020). Empobrecimiento de los hogares y cambios en el abastecimiento de alimentos por al COVID-19 en Lima, Perú. Revista electrónica de recursos en internet sobre geografía y ciencias sociales Ar@cne, 24(243), 1-23. Recuperado de https://revistes.ub.edu/index.php/aracne/article/view/31627/31614 INEI. (2019). Perú: Evolución de los indicadores de empleo e ingreso por departamento, 2007-2018. Lima: INEl. Recuperado de https://www.inei.gob.pe/media/ 
MenuRecursivo/publicaciones_digitales/Est/Lib1678/libro.pdf

Lozano, L., Lozano, S. \& Robledo, R. (2020). Desempleo en tiempos de COVID- 19: Efectos socioeconómicos en el entorno familiar. Journal of science and research, 5(4), 187-197. Recuperado de https://revistas.utb.edu.ec/index.php/sr/article/ view/926/667

Martínez, D., Bucio, C., \& Ortíz, E. (2021). Cópulas dinámicas en el índice de morosidad del crédito al consumo en México. Lumina: Revista iberoamericana de contabilidad, administración y economía, 22, 1-20. Recuperado de https://revistasum.umanizales. edu.co/ojs/index.php/Lumina/article/view/4132/6438

Narváez, A. (2020). Deuda financiera y quiebra de familias: el caso peruano. Revista de Investigaciones $U L C B, 7(1), 109-124$. Recuperado de https://revistas.ulcb.edu.pe/ index.php/REVISTAULCB/article/view/172/329

Parada, D., \& Zambrano, G. (2020). Reinvención de la vida cotidiana en mujeres cucuteñas en tiempos de COVID-19. Psicoperspectivas individuo y sociedad, 19(3), 1-11. Recuperado de https://scielo.conicyt.cl/pdf/psicop/v19n3/0718-6924psicop-19-03-41.pdf

Rodríguez, J. (2021). Situación económica, política fiscal y pandemia en Colombia. Revista de Economía Institucional, 22(43), 249-263. Recuperado de http://www.scielo. org.co/pdf/rei/v23n44/0124-5996-rei-23-44-249.pdf

SBS. (2020). Informe de estabilidad del sistema financiero (Informe diciembre 2020). Recuperado de https://www.sbs.gob.pe/Portals/0/jer/pub_InformeEstabilidad/ Informe\%20de\%20Estabilidad\%20Financiera_2020_II.pdf

Sociedad de Comercio Exterior del Perú.(2020). Los persistentes problemas del mercado laboral generarán 1.5 millones de personas desempleadas al finalizarel año. Recuperado de https://www.comexperu.org.pe/articulo/los-persistentes-problemas-delmercado-laboral-generaran-15-millones-de-personas-desempleadas-al-finalizarel-ano

Fecha de recepción: 15/03/2021 Fecha de aceptación: 21/04/2021 Correspondencia: asesorvitucho@gmail.com 